\title{
Is it all Lynch syndrome?: An assessment of family history in individuals with mismatch repair-deficient tumors
}

\author{
Katherine M. Dempsey, MS ${ }^{1-3}$, Russell Broaddus, MD, PhD ${ }^{3,4}$, Y. Nancy You, MD5, \\ Sarah Jane Noblin, MS ${ }^{3,6,7}$, Maureen Mork, MS 1,5 , Bryan Fellman, $\mathrm{MS}^{8}$, Diana Urbauer, $\mathrm{MS}^{8}$, \\ Molly Daniels, $\mathrm{MS}^{1,2,3,7}$ and Karen Lu, MD ${ }^{1,3}$
}

\begin{abstract}
Purpose: Mismatch repair-deficient (MMRD) colorectal cancer (CRC) and endometrial cancer (EC) may be suggestive of Lynch syndrome (LS). LS can be confirmed only by positive germ-line testing. It is unclear if individuals with MMRD tumors but no identifiable cause (MMRD+/germ-line-) have LS. Because LS is hereditary, individuals with LS are expected to have family histories of LS-related tumors. Our study compared the family histories of MMRD+/germ-line- CRC and/or EC patients with LS CRC and/ or EC patients.
\end{abstract}

Methods: A total of 253 individuals with an MMRD CRC or EC from one institution were included for analysis in one of four groups: LS; MMRD+/germ-line-; MMRD tumor with variant of uncertain significance (MMRD+/VUS); and sporadic MSI-H (MMRD tumor with $M L H 1$ promoter hypermethylation or BRAF mutation). Family histories were analyzed utilizing MMRpro and
PREMM $_{1,2,6}$. Kruskal-Wallis tests were used to compare family history scores.

Results: MMRD+/germ-line- individuals had significantly lower median family history scores $\left(\right.$ MMRpro $=8.1$, PREMM $\left._{1,2,6}=7.3\right)$ than did LS individuals (MMRpro $=89.8$, PREMM $_{1,2,6}=26.1, P<0.0001$ ).

Conclusion: MMRD+/germ-line- individuals have less suggestive family histories of LS than individuals with LS. These results imply that MMRD+/germ-line- individuals may not all have LS. This finding highlights the need to determine other causes of MMRD tumors so that these patients and their families can be accurately counseled regarding screening and management.

Genet Med advance online publication 23 October 2014

Key Words: genetic testing; Lynch syndrome; mismatch repair-deficient tumor; tumor studies

\section{INTRODUCTION}

Lynch syndrome (LS), also known as hereditary nonpolyposis colorectal cancer, is an autosomal dominant hereditary cancer syndrome that confers a significantly increased lifetime risk of colorectal cancer (CRC) and endometrial cancer (EC), as well as an increased risk of a number of other cancers. ${ }^{1-5}$ LS accounts for $2-4 \%$ of CRCs ${ }^{6}$ and $~ 2 \%$ of ECs. ${ }^{7}$ It is caused by germ-line mutations in the mismatch repair (MMR) genes MLH1, MSH2, MSH6, and PMS2, as well as EPCAM/TACSTD $1 .{ }^{8,9}$

Tumor studies, consisting of microsatellite instability (MSI) and immunohistochemistry (IHC) studies, have proven to be effective at identifying individuals at risk for having LS. A universal tumor testing approach has been shown to have a sensitivity of up to $100 \%$ and a specificity of up to $93 \% .{ }^{10}$ Approximately 95\% of LS-related CRCs are found to be MSIhigh (MSI-H). Loss of staining on IHC of one or more proteins is indicative of a somatic or germ-line defect in the MMR genes. Tumors that exhibit high MSI and/or loss of staining on IHC are considered to be MMR-deficient (MMRD) and warrant further investigation.

In the case of a tumor that is MSI-H with loss of MLH1 and PMS2 on IHC, sporadic causes, including MLH1 hypermethylation and/or a BRAF V600E mutation in CRC and MLH1 hypermethylation in EC, must be ruled out. BRAF V600E mutations are indicative of $M L H 1$ hypermethylation in individuals with CRC. BRAF V600E mutations are not related to MLH1 hypermethylation in individuals with EC. Individuals with MMRD tumors lacking these known sporadic causes should undergo LS germ-line testing, including sequencing and deletion/duplication analysis of the appropriate MMR gene(s), which, if positive, confirms a diagnosis of LS.

As tumor studies have become more widespread, there is an emerging cohort of individuals who have MMRD tumors lacking known sporadic causes ( $M L H 1$ promoter hypermethylation and/or BRAF V600E mutations in CRC; MLH1 promoter hypermethylation in EC) and no identifiable mutation (MMRD+/germ-line-). For example, in a recent study 23 of 59

${ }^{1}$ Department of Clinical Cancer Genetics, University of Texas MD Anderson Cancer Center, Houston, Texas, USA; ${ }^{2}$ Department of Gynecologic Oncology and Reproductive Medicine, University of Texas MD Anderson Cancer Center, Houston, Texas, USA; ${ }^{3}$ The University of Texas Graduate School of Biomedical Sciences at Houston, Houston, Texas, USA; ${ }^{4}$ Department of Pathology Administration, University of Texas MD Anderson Cancer Center, Houston, Texas, USA; ${ }^{5}$ eepartment of Surgical Oncology, University of Texas MD Anderson Cancer Center, Houston, Texas, USA; ${ }^{6}$ Department of Obstetrics and Gynecology, The University of Texas Health Science Center at Houston, Houston, Texas, USA; ${ }^{7}$ Department of Pediatrics, University of Texas Health Science Center at Houston, Houston, Texas, USA; ${ }^{8}$ Department of Biostatistics, University of Texas MD Anderson Cancer Center, Houston, Texas, USA. Correspondence: KarenLu (khlu@mdanderson.org) 
patients (38.9\%) with MMRD tumors lacking known sporadic cause who pursued genetic testing had uninformative negative genetic test results. ${ }^{11}$ It is unclear if these individuals truly have LS. There are two possible explanations as to why this cohort has emerged. The first is that these individuals do have LS, but our current genetic testing technology is not sensitive enough to detect the germ-line mutations in these individuals. There are other documented rare heritable causes of LS, including constitutional MLH1 hypermethlation and complex rearrangements of the MMR genes that cannot currently be detected by clinically available genetic testing technology. ${ }^{12}$ The second possible explanation is that these individuals do not have LS and there is another explanation for the MMRD tumor phenotype, such as epigenetic or somatic changes or modifier genes. In addition to MLH1 hypermethylation and BRAF V600E mutations, it has recently been discovered that biallelic somatic mutations in the MMR genes are also possible ${ }^{13,14}$ and can account for up to 50\% of unexplained MMRD tumors. ${ }^{15}$ In addition, although rare, somatic mosaicism has been observed. ${ }^{14}$

From a clinical standpoint, a family history can be a very powerful risk-assessment tool and can either significantly increase the concern or significantly decrease the concern for a hereditary cancer syndrome. Because LS is a hereditary condition, we would generally expect individuals with LS to have a family history of LS-related cancers. Therefore, to examine the likelihood of LS in MMRD+/germ-line- individuals, we analyzed and compared the family histories of $\mathrm{MMRD}+/$ germline-individuals, individuals with LS, individuals with MMRD tumor with variants of uncertain significance (MMRD+/VUS), and individuals with sporadic MSI-H to determine if there was a difference in suggestiveness of family history between the groups.

\section{MATERIALS AND METHODS}

\section{Patient and data collection}

The study sample included probands who presented to the University of Texas MD Anderson Cancer Center for genetic counseling for MMRD CRC and/or EC from January 1995 to October 2012. Personal and tumor-related information was collected for all probands from the MD Anderson electronic medical record. Pedigrees for all probands were obtained from the Clinical Cancer Genetics MD Anderson database. Individuals were excluded if tumor study results, germ-line testing results, or a pedigree were not available. Individuals with tumor studies performed only on tissues other than colon or endometrium were excluded. MMRD tumors were defined as one of the following: MS-stable with loss of staining on IHC; MSI-H with staining intact on IHC; or MSI-H with loss of staining on IHC. Individuals who were MS-stable with intact staining were not included in the initial query of the database. If multiple members of a family were seen for genetic counseling, only the individual who presented initially was included for analysis. Individuals who had a personal or family history indicative of another hereditary cancer syndrome were excluded. Individuals with tumors that were MSI-low and normal IHC who underwent germ-line testing are not included in statistical analysis because of the lack of consensus that these tumor study results should be considered suggestive of LS and the lack of consistent referral of this patient population for genetic counseling and testing. The study protocol was approved by the University of Texas MD Anderson Cancer Center on 1 November 2012.

\section{Risk-assessment models}

Pedigree information was quantified utilizing both PREMM 1,2,6 $_{1}$ (available through the Dana Farber Cancer Institute; http:// premm.dfci.harvard.edu/) and MMRpro 5.1 (available through University of Texas Southwestern's CancerGene Version 5.1; http://www4.utsouthwestern.edu/breasthealth/cagene/). Both models are clinically validated risk-assessment tools that provide the likelihood of identifying a germ-line mutation in one of the MMR genes (MLH1, MSH2, or MSH6) in the proband by taking into account personal and family history information.

If exact ages of family members were not available, conservative estimation based on available information was utilized. Individuals for whom limited information was available were excluded from pedigree analysis. Half-siblings were not used in pedigree analysis. MMRpro input can include MSI and IHC results but not MLH1 hypermethylation and/or BRAF V600E mutation results. MMRpro has been validated for use both with and without inclusion of tumor study results. ${ }^{16}$ Because everyone in the study population has abnormal tumor study results, and because our purpose in calculating the MMRpro score was to summarize the suggestiveness of the personal and family history without reference to tumor study results, we chose to not include the tumor study results when running MMRpro. Additionally, PREMM $_{1,2,6}$ does not incorporate tumor study results.

\section{Statistical analysis}

Summary statistics were performed to analyze the demographic, clinical, and genetic characteristics of the patients. A $\chi^{2}$ test, Fisher's exact test, or Kruskal-Wallis test was conducted to compare demographic characteristics between germ-line testing groups, and pairwise comparisons were performed to determine statistical significance between groups. To control for multiple comparisons, a Bonferroni correction was used, with $\alpha$ defined as 0.008 . A Kruskal-Wallis test was conducted to compare family history scores. A Wilcoxon rank-sum test was conducted to compare all pairwise comparisons. To control for multiple comparisons, a Bonferroni correction was used for the pairwise comparisons when statistical significance was defined at the $\alpha$ $=0.008$ level. Wilcoxon rank-sum tests were also conducted to compare germ-line testing groups by colon/endometrial cancer, gender, and age. For these pairwise comparisons, a Bonferonni correction was used when statistical significance was defined at the $\alpha=0.01$ level. A logistic regression model was also conducted with a term for family history as a predictor to estimate the odds of testing germ-line positive. The model controlled for the following variables: age, gender, cancer type, and ethnicity. All analyses were performed using STATA/SE 12.1. 


\section{RESULTS}

The information for 274 individuals was collected. These individuals were classified into one of four groups: LS, MMRD+/ germ-line-, MMRD+/VUS, and known sporadic (sporadic MSI-H), defined as tumors with the presence of MLH1 hypermethylation and/or BRAF V600E mutation in CRC and MLH1 hypermethylation in EC. Twenty-one individuals with MSI-low tumors with intact IHC were excluded from further analysis. Of the final population of 253 individuals with MMRD tumors, 97 (80 with CRC, 12 with EC, 5 with both) had a mutation identified on germ-line testing (38.3\%), 70 (58 with CRC, 12 with EC) had no mutation identified on germ-line testing and no sporadic cause identified (27.7\%), 31 (28 CRC, $3 \mathrm{EC}$ ) were found to have VUS upon germ-line testing (12.3\%), and 55 (40 CRC, 15 EC) were found to have a sporadic tumor (21.7\%).

\section{Demographic, clinical, and pathological characteristics}

Demographic information for the final population $(n=253)$ is summarized in Table 1. Statistically significant differences between groups $(\alpha=0.05)$ were identified for age at cancer diagnosis $(P=<0.0001)$, ethnicity $(P=0.0402)$, and type of cancer $(P=0.0476)$. Overall, the mean age of diagnosis was 51.5 years $(\mathrm{SD}=13.2)$. Average age of diagnosis was 48.3 years $(\mathrm{SD}=$ 12.6) for the LS group, 51.3 years $(S D=12.7)$ for the $M M R D+/$ germ-line- group, and 46.2 years $(\mathrm{SD}=8.9)$ for the $\mathrm{MMRD}+/$ VUS group, all significantly younger than that of the sporadic MSI-H group (average age of diagnosis $=60.5$ years, $\mathrm{SD}=13.0$, $P<0.008)$.

Tumor characteristics for individuals with CRC overall $(n=$ 211) and between groups (LS, $n=85$; MMRD+/germ-line-,$n=$ 58 ; MMRD+/VUS, $n=28$; sporadic MSI- $\mathrm{H}, n=40$ ) are summarized in Table 2. Statistical significance was reached for gender $(P=0.0228)$ and additional polyps identified at time of diagnosis $(P=0.0034) ; 64.7 \%$ of the overall population $(n=116)$ had no additional polyps at the time of diagnosis. Individuals in the LS group were more likely to have additional polyps at the time of cancer diagnosis (47\%) than those in the sporadic MSI-H group $(P<0.008)$, and individuals in the MMRD+/VUS group were significantly less likely to have additional polyps at the time of diagnosis $(9.1 \%)$ than those in the LS group $(P<0.008)$.

Table 1 Demographic information

\begin{tabular}{|c|c|c|c|c|c|c|c|c|c|c|c|}
\hline & \multicolumn{10}{|c|}{ Germ-line testing group } & \multirow[b]{3}{*}{$P$ value } \\
\hline & \multicolumn{2}{|c|}{$\begin{array}{c}\text { LS } \\
(n=97)\end{array}$} & \multicolumn{2}{|c|}{$\begin{array}{c}\text { MMRD+/Lynch- } \\
(n=70)\end{array}$} & \multicolumn{2}{|c|}{$\begin{array}{l}\text { MMRD+/VUS } \\
(n=31)\end{array}$} & \multicolumn{2}{|c|}{$\begin{array}{l}\text { Sporadic MSI-H } \\
\quad(n=55)\end{array}$} & \multicolumn{2}{|c|}{$\begin{array}{c}\text { Total } \\
(n=253)\end{array}$} & \\
\hline & $N$ & $\%$ & $N$ & $\%$ & $N$ & $\%$ & $N$ & $\%$ & $N$ & $\%$ & \\
\hline$N$ & 97 & & 70 & & 31 & & 55 & & 253 & & \\
\hline Mean (SD) & $48.3(12.6)$ & & $51.3(12.7)$ & & $46.2(8.9)$ & & $60.5(13.0)$ & & $51.5(13.2)$ & & \\
\hline Alive & 79 & 81.4 & 62 & 88.6 & 28 & 90.3 & 48 & 87.3 & 217 & 85.8 & \\
\hline Deceased & 18 & 18.6 & 8 & 11.4 & 3 & 9.7 & 7 & 12.7 & 36 & 14.2 & \\
\hline Ethnicityc & & & & & & & & & & & 0.0402 \\
\hline Caucasian & 77 & 80.2 & 57 & 81.4 & 18 & 58.1 & 49 & 89.1 & 201 & 79.8 & \\
\hline African American & 6 & 6.3 & 4 & 5.7 & 4 & 12.9 & 1 & 1.8 & 15 & 6.0 & \\
\hline Tumor location & & & & & & & & & & & 0.0476 \\
\hline Colon & 80 & 82.5 & 58 & 82.9 & 28 & 90.3 & 40 & 72.7 & 206 & 81.4 & \\
\hline Endometrial & 12 & 12.4 & 12 & 17.1 & 3 & 9.7 & 15 & 27.3 & 42 & 16.6 & \\
\hline Both & 5 & 5.2 & 0 & 0.0 & 0 & 0.0 & 0 & 0.0 & 5 & 2.0 & \\
\hline Other cancer & & & & & & & & & & 0.1498 & \\
\hline No & 50 & 51.5 & 47 & 67.1 & 20 & 64.5 & 36 & 65.5 & 153 & 60.5 & \\
\hline Yes & 47 & 48.5 & 23 & 32.9 & 11 & 35.5 & 19 & 34.5 & 100 & 39.5 & \\
\hline \multicolumn{12}{|c|}{ Number of other cancers } \\
\hline 0 & 50 & 51.5 & 47 & 67.1 & 20 & 64.5 & 36 & 65.5 & 153 & 60.5 & \\
\hline 1 & 26 & 26.8 & 16 & 22.9 & 9 & 29.0 & 12 & 21.8 & 63 & 24.9 & \\
\hline
\end{tabular}

LS, Lynch syndrome; Max, maximum; Med, median; Min, minimum; MMRD, mismatch repair-deficient; MSI-H, microsatellite instability-high; VUS, variant of uncertain significance.

Significant pairwise comparisons $(\alpha<0.008)$ : a LS versus sporadic MSI-H; ${ }^{b} M M R D+/ g e r m-l i n e-$ versus sporadic MSI-H; ${ }^{c} M M R D+N U S$ versus sporadic MSI-H. 
Tumor characteristics for individuals with EC overall ( $n=47)$ and between groups (LS, $n=17$; MMRD+/germline-, $n=12$; MMRD+/VUS, $n=3$; sporadic MSI-H, $n=$ $15)$ are summarized in Table 3 . There was a significant difference between groups for location of tumor $(P=0.0407)$. Overall, the majority of tumors were located in the uterine body (78.7\%). All sporadic MSI-H tumors were located in the uterine body. There was also a significant difference between the IHC results of the various groups $(P=0.002)$. Overall, IHC revealed loss of MLH1 and PMS2 in 23 tumors (48.9\%) and loss of MSH2 and MSH6 in 16 tumors (34.0\%). In general, the LS group had more tumors with loss of hMSH2 and hMSH6 (58.8\%), as well as of hMSH6 only (11.8\%). As expected, the sporadic MSI-H group had significantly more individuals with loss of MLH1/PMS2 on IHC (100\%) than the other groups $(P<0.008)$. Average body mass index $(\mathrm{BMI})$ of individuals with $\mathrm{EC}$ varied significantly between groups $(P$ $=0.0138)$. Overall average BMI for the EC group was 28.8. Individuals in the LS group, on average, had a BMI of 23.7, which was lower than that of the overall group and the three

Table 2 Colorectal cancer characteristics

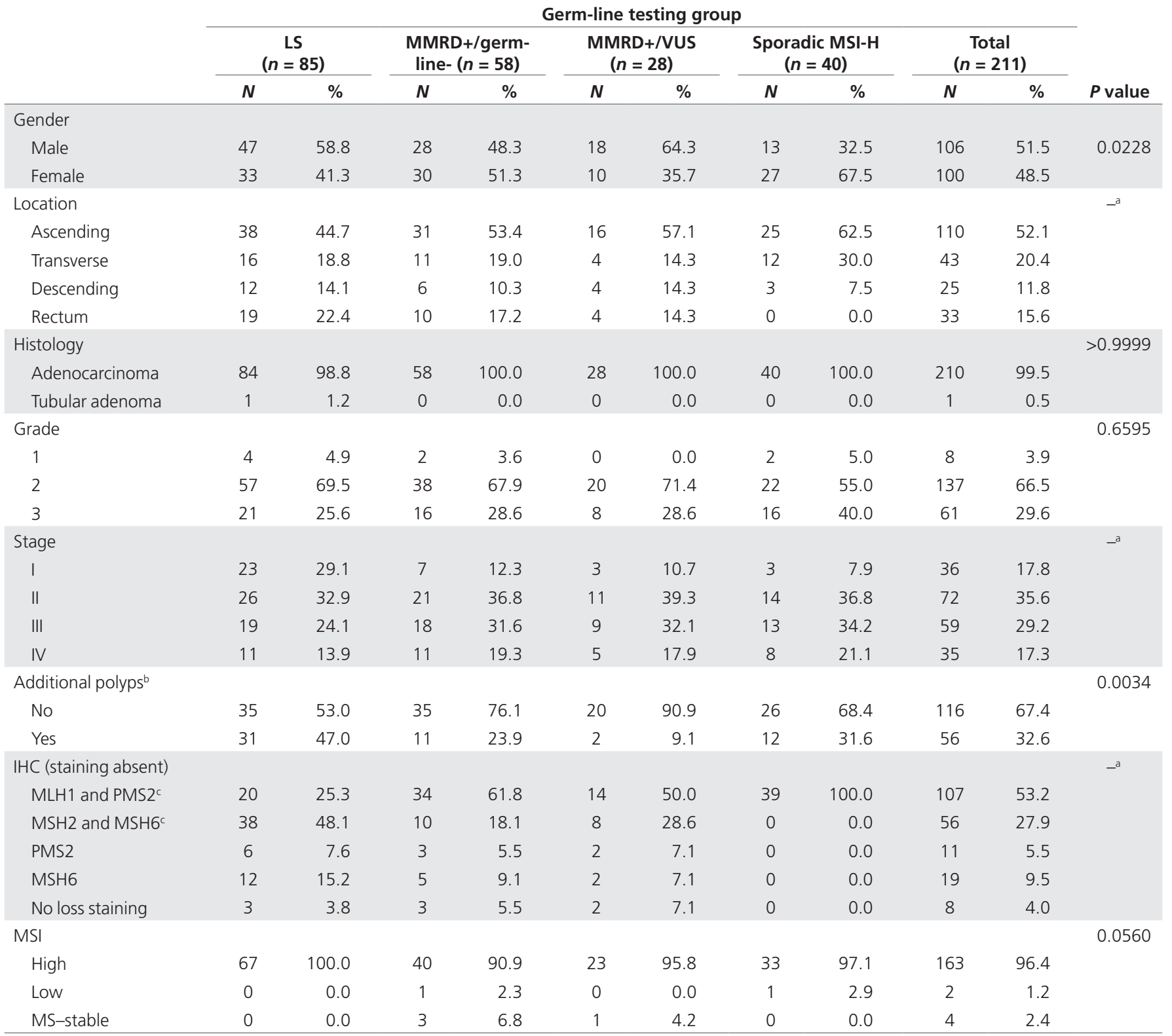

IHC, immunohistochemistry; LS, Lynch syndrome; MMRD, mismatch repair-deficient; MSI-H, microsatellite instability-high; VUS, variant of uncertain significance. astatistical significance $(\alpha<0.008)$ could not be established because numerous individuals were missing this demographic characteristic. ${ }^{b}$ Significant pairwise comparisons $(\alpha<0.008)$ : LS versus MMRD+NUS. There were 21 individuals overall (5 LS, 9 MMRD+/germ-line-, 1 MMRD+/NUS, and 6 sporadic MSI-H) who had loss of staining for MLH1 alone and 1 individual overall (MMRD+/germ-line-) who had loss of staining for MSH2 alone before staining for PMS2 and MSH6 was available. These individuals were incorporated into the statistics for MLH1/PMS2 and MSH2/MSH6 because their samples would likely have stained negative for PMS2 and MSH6 if the tests had been available. 
other groups (MMRD+/germ-line- $=30.5 \mathrm{BMI} ; \mathrm{MMRD}+$ / VUS = 26.8 BMI; sporadic MSI-H = 31.9 BMI).

\section{Germ-line testing}

Of the 97 individuals identified as having a mutation in one of the MMR genes, 26 had mutations in MLH1 (26.8\%), 45 had mutations in $\mathrm{MSH} 2$ (46.4\%), 18 had mutations in MSH6 (18.6\%), 7 had mutations in PMS2 (9.3\%), and 1 had a mutation in EPCAM (1.0\%). Of the 31 individuals identified as having VUS in one of their MMR genes, 16 had VUS in MLH1 (51.6\%), 12 had VUS in MSH2 (38.7\%), 2 had VUS in MSH6 (6.5\%), and 1 had VUS in PMS2 (3.2\%).

\section{Family history assessment}

Median family history scores of LS, MMRD+/germ-line-, MMRD+/VUS, and sporadic MSI-H were assessed and compared (Figure 1). The median family history scores were 89.8 (range, 0.0-100) for MMRpro and 26.1 (range, 5.0-97.6) for PREMM $_{1,2,6}$ for the LS group, 8.1 (range, 0.0-100) for MMRpro and 7.3 (range, 5.0-93.1) for PREMM PR.,6 $_{1,6}$ for the MMRD+/ germ-line- group, 28.0 (range, 0.0-99.8) for MMRpro and 11.1 (range, 5.0-82.5) for PREMM ${ }_{1,2,6}$ for the MMRD+/VUS group, and 0.7 (range, 0.0-94.0) for MMRpro and 5.0 (range, 5.0-37.4) for PREMM ${ }_{1,2,6}$ for the sporadic MSI-H group. In light of the reduced penetrance in MSH6-associated and PMS2-associated LS as compared with $M L H 1$-associated and $M S H 2$-associated LS, the LS group also was split into MLH1/MSH2-positive and MSH6/PMS2-positive groups. The median family history scores were 95.1 (range, 0.3-100.0) for MMRpro and 38.7 (range, 5.097.6) for PREMM ${ }_{1,2,6}$ for the MLH1/MSH2-positive group, and 7.7 (range, 0.0-92.1) for MMRpro and 7.3 (range, 5.0-65.0) for PREMM $_{12,6}$ for the MSH6/PMS2-positive group.

The overall LS group had significantly higher family history scores on both MMRpro and PREMM ${ }_{1,2,6}$ than did the

Table 3 Endometrial cancer characteristics

\begin{tabular}{|c|c|c|c|c|c|c|c|c|c|c|c|}
\hline & \multicolumn{10}{|c|}{ Germ-line testing group } & \multirow[b]{3}{*}{$P$ value } \\
\hline & \multicolumn{2}{|c|}{$\begin{array}{c}\text { LS } \\
(n=17)\end{array}$} & \multicolumn{2}{|c|}{$\begin{array}{l}\text { MMRD+/germ- } \\
\text { line- }(n=12)\end{array}$} & \multicolumn{2}{|c|}{$\begin{array}{c}\text { MMRD+/VUS } \\
(n=3)\end{array}$} & \multicolumn{2}{|c|}{$\begin{array}{l}\text { Sporadic MSI-H } \\
\quad(n=15)\end{array}$} & \multicolumn{2}{|c|}{$\begin{array}{c}\text { Total } \\
(n=47)\end{array}$} & \\
\hline & $N$ & $\%$ & $N$ & $\%$ & $N$ & $\%$ & $N$ & $\%$ & $N$ & $\%$ & \\
\hline Location & & & & & & & & & & & 0.0407 \\
\hline Lower uterine & 6 & 35.3 & 3 & 25.0 & 1 & 33.3 & 0 & 0.0 & 10 & 21.3 & \\
\hline Uterine body & 11 & 64.7 & 9 & 75.0 & 2 & 66.7 & 15 & 100.0 & 37 & 78.7 & \\
\hline Histology & & & & & & & & & & & 0.1112 \\
\hline Endometrioid & 14 & 82.4 & 10 & 83.3 & 2 & 66.7 & 14 & 93.3 & 40 & 85.1 & \\
\hline Serous & 1 & 5.9 & 0 & 0.0 & 0 & 0.0 & 0 & 0.0 & 1 & 2.1 & \\
\hline Mixed high & 2 & 11.8 & 0 & 0.0 & 0 & 0.0 & 1 & 6.7 & 3 & 6.4 & \\
\hline Clear-cell & 0 & 0.0 & 2 & 16.7 & 0 & 0.0 & 0 & 0.0 & 2 & 4.3 & \\
\hline Papillary & 0 & 0.0 & 0 & 0.0 & 1 & 33.3 & 0 & 0.0 & 1 & 2.1 & \\
\hline Grade & & & & & & & & & & & 0.9867 \\
\hline 1 & 3 & 20.0 & 2 & 18.2 & 0 & 0.0 & 2 & 13.3 & 7 & 16.3 & \\
\hline 2 & 9 & 60.0 & 7 & 63.6 & 2 & 100.0 & 11 & 73.3 & 29 & 67.4 & \\
\hline 3 & 3 & 20.0 & 2 & 18.2 & 0 & 0.0 & 2 & 13.3 & 7 & 16.3 & \\
\hline Stage & & & & & & & & & & & 0.2890 \\
\hline I & 7 & 53.8 & 5 & 55.6 & 0 & 0.0 & 8 & 61.5 & 20 & 52.6 & \\
\hline$\|$ & 2 & 15.4 & 0 & 0.0 & 2 & 66.7 & 2 & 15.4 & 6 & 15.8 & \\
\hline III & 3 & 23.1 & 4 & 44.4 & 1 & 33.3 & 2 & 15.4 & 10 & 26.3 & \\
\hline IV & 1 & 7.7 & 0 & 0.0 & 0 & 0.0 & 1 & 7.7 & 2 & 5.3 & \\
\hline $\mathrm{IHC}^{\mathrm{a}}$ (staining absent) & & & & & & & & & & & 0.0002 \\
\hline MLH1 and PMS2 ${ }^{\mathrm{b}}$ & 3 & 17.6 & 7 & 58.3 & 1 & 33.3 & 15 & 100.0 & 26 & 55.3 & \\
\hline MSH2 and MSH6 ${ }^{b}$ & 10 & 58.8 & 4 & 33.3 & 2 & 66.7 & 0 & 0.0 & 16 & 34.0 & \\
\hline PMS2 & 1 & 5.9 & 0 & 0.0 & 0 & 0.0 & 0 & 0.0 & 1 & 2.1 & \\
\hline MSH6 & 2 & 11.8 & 0 & 0.0 & 0 & 0.0 & 0 & 0.0 & 2 & 4.3 & \\
\hline No loss of staining & 1 & 5.9 & 1 & 8.3 & 0 & 0.0 & 0 & 0.0 & 2 & 4.3 & \\
\hline MSI & & & & & & & & & & & 0.8603 \\
\hline High & 9 & 90.0 & 8 & 88.9 & 3 & 100.0 & 11 & 91.7 & 31 & 91.2 & \\
\hline Low & 1 & 10.0 & 0 & 0.0 & 0 & 0.0 & 1 & 8.3 & 2 & 5.9 & \\
\hline MS-stable & 0 & 0.0 & 1 & 11.1 & 0 & 0.0 & 0 & 0.0 & 1 & 2.9 & \\
\hline
\end{tabular}

IHC, immunohistochemistry; LS, Lynch syndrome; MMRD, mismatch repair-deficient; MSI-H, microsatellite instability-high; VUS, variant of uncertain significance.

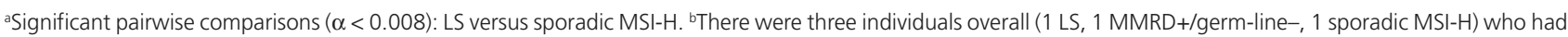
staining performed for MLH1 only before staining for PMS2 was available. These individuals have been added to the statistics for MLH1/PMS2 because their samples likely would have stained negative for PMS2 if the test had been available. 
MMRD+/germ-line- group (MMRpro: $P<0.0001 ;$ PREMM $_{1,2,6}$ : $P<0.0001$ ), the MMRD+/VUS group (MMRpro: $P=0.0063$; PREMM $_{1,2,6}: P=0.0038$ ), and the sporadic MSI-H group (MMRpro: $P<0.0001$ PREMM $_{1,2.6}: P<0.0001$ ). The MMRD+/ germ-line- group had significantly higher median family history scores on both MMRpro and PREMM ${ }_{1,2,6}$ than did the sporadic MSI-H group (MMRpro: $P<0.0001$; PREMM $_{1,2,6}$ : $P<0.0001)$. There was no significant difference between the MMRD+/germ-line- group and the MMRD+/VUS group for either MMRpro $(P=0.1924)$ or $\operatorname{PREMM}_{1,2,6}(P=0.0249)$.
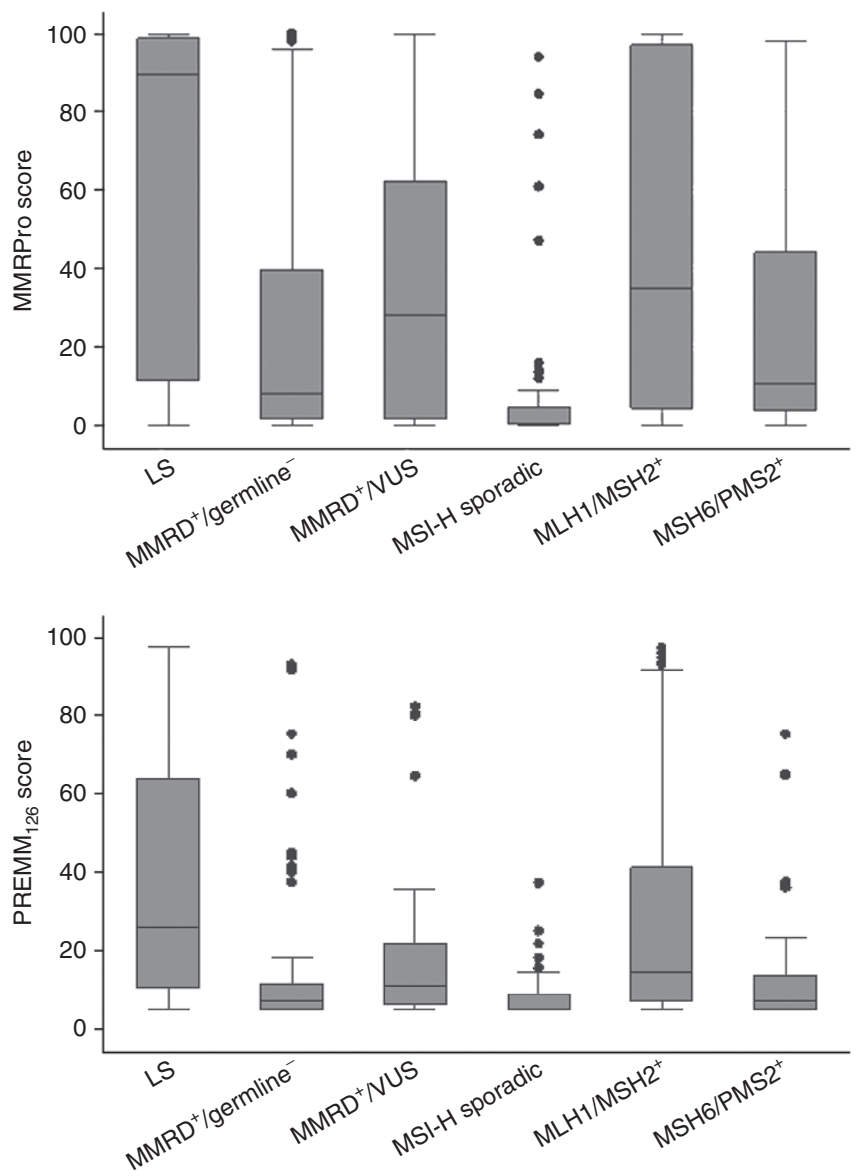

Figure 1 Median family history scores.
The MMRD+/VUS group had significantly higher family history scores than the sporadic MSI-H group for both MMRpro $(P<0.0001)$ and PREMM $_{1,2,6}(P<0.0001)$. When the LS group was split into MLH1/MSH2-positive and MSH6/PMS2-positive groups and compared with the MMRD+/germ-line- group, it was observed that the MLH1/MSH2-positive group had significantly higher family history scores than the MMRD+/germline- group (MMRpro: $P<0.001$ PREMM $_{1,2,6}: P<0.001$ ). The MSH6/PMS2-positive group had family history scores similar to those of the MMRD+/germ-line- group (MMRpro: $P=$ 0.5933; PREMM $_{1,2,6}: P=0.6938$ ).

When the family history scores were compared by gene implicated for the LS group and the MMRD+/germ-linegroup, it was observed that the difference between these two groups was driven by the family history scores of individuals with $M L H 1$ and MSH2 mutations (Table 4). There was a significant difference between the family history scores of LS individuals with an $\mathrm{MLH1}$ mutation versus those of MMRD+/ germ-line- individuals with loss of MLH1/PMS2 on IHC for both MMRpro $(P<0.0001)$ and PREMM $1,2,6(P<0.0001)$. The difference between the LS group with $M S H 2$ mutations and the MMRD+/germ-line- group with loss of MSH2/MSH6 on IHC was trending toward significance for both MMRpro $(P=$ 0.0812) and $\operatorname{PREMM}_{1,2,6}(P=0.0536)$.

Family history scores were also compared based on cancer site. There was no significant difference in the family history scores between the CRC LS and EC LS (MMRpro: $P=0.3446$; PREMM $_{1,2,6}: P=0.3130$ ), CRC MMRD+/germ-line- and EC MMRD+/germ-line- (MMRpro: $P=0.1878 ;$ PREMM $_{1,2,6^{\circ}}$ : $P=0.6832)$, or CRC MMRD+/VUS and EC MMRD+/VUS (MMRpro: $P=0.6884$; PREMM $_{1,2,6}: P=0.7888$ ) groups. Individuals with $\mathrm{EC}$ in the KS group had significantly higher family history scores than individuals with CRC in the KS group on both MMRpro $(P=0.0039)$ and $\operatorname{PREMM}_{1,2,6}(P=0.0016)$.

Both family history modalities were significant predictors of testing germ-line positive in the logistic regression analysis. For each one-unit increase in MMRpro, the odds of being germline positive increased by a factor of 1.02 (95\% confidence interval: $1.01-1.03 ; P<0.001)$. For each one-unit increase in PREMM $_{12,6}$ the odds of being germ-line positive increased by a factor of 1.04 (95\% confidence interval: 1.02-1.05; $P=<0.001$ ).

Table 4 LS family history scores versus MMRD+/germ-line- family history scores by gene implicated

\begin{tabular}{|c|c|c|c|c|c|c|c|c|c|c|c|}
\hline \multirow[b]{2}{*}{ Group } & \multirow[b]{2}{*}{ Gene implicated } & \multicolumn{5}{|c|}{ MMRpro } & \multicolumn{5}{|c|}{ PREMM $_{1,2,6}$} \\
\hline & & $N$ & Min & Med & Max & $P$ value & $N$ & Min & Med & Max & $P$ value \\
\hline LS & MLH1 & 26 & 4.3 & 98.4 & 99.8 & $<0.0001$ & 26 & 5.5 & 49.3 & 96.0 & $<0.0001$ \\
\hline MMRD+/germ-line- & MLH1/PMS2 & 31 & 0 & 6.9 & 87.3 & & 31 & 5 & 5.6 & 45 & \\
\hline LS & MSH2 & 45 & 0.3 & 90.8 & 100.0 & 0.0812 & 45 & 0.1 & 36.7 & 100.0 & 0.0536 \\
\hline MMRD+/germ-line- & MSH2/MSH6 & 13 & 0.1 & 15.8 & 100.0 & & 13 & 5.0 & 12.1 & 91.8 & \\
\hline LS & MSH6 & 18 & 0.3 & 7.3 & 91.7 & 0.9406 & 18 & 5.0 & 8.1 & 65.0 & 0.5000 \\
\hline MMRD+/germ-line- & PMS2 & 3 & 20.6 & 96.0 & 98.1 & & 3 & 12.6 & 37.6 & 75.4 & \\
\hline
\end{tabular}

LS, Lynch syndrome; Max, maximum; Med, median; Min, minimum; MMRD, mismatch repair-deficient. 


\section{DISCUSSION}

Individuals with MMRD+/germ-line- tumors have significantly lower median family history scores than individuals with LS, indicating that they have family histories that are less suggestive of a hereditary cancer syndrome. Based on these results, and on the growing body of literature surrounding MMRD tumors, it is plausible to consider that these individuals have a disease distinct from classic LS. Tumor studies may not be considered diagnostic of LS in the absence of an unidentifiable germ-line mutation, particularly when family history is entirely absent. Interestingly, individuals with MMRD+/germ-linetumors have significantly higher median family history scores than individuals with an MSI-H sporadic tumor, suggesting that it would also be incorrect to assume that all MMRD+/germline- tumors are secondary to sporadic or epigenetic causes.

In addition, other data support the notion that MMRD+/ germ-line tumors may be due to a combination of hereditary and sporadic causes. EC patients with LS had an average BMI in the normal range, whereas the remaining three groups had average BMI scores in the overweight to obese range. Obesity is a known risk factor for sporadic EC. Therefore, the high BMI scores in the MMRD+/germ-line- group, similar to what was seen in the sporadic group, indicate the possibility of sporadic causes for the MMRD+/germ-line- ECs. In addition, individuals in the overall MMRD+/germ-line- group had an average age of diagnosis that was similar to the average age of diagnosis in the LS group. The average age of diagnosis was significantly younger than that of the sporadic MSI-H group and younger than what has been observed in the general population. ${ }^{17}$ It was similar to what has been described as the average age of LS-related cancer onset in the literature, ${ }^{18}$ suggesting the possibility of a hereditary component for the MMRD+l germ-line- group.

MMRD+/germ-line- individuals lie in the middle of the family history spectrum. Possible explanations as to why this is so could be that the cohort could be a mixture of individuals with true LS and in whom our current genetic testing technology is not sensitive enough to detect their mutation, or that these individuals do not have LS but instead have an MMRD sporadic tumor. It is also possible that this cohort represents a currently undefined hereditary cancer syndrome or subset of LS with lower cancer risks than true LS but increased risks compared with the general population.

Within the LS group, individuals with an $\mathrm{MLH1}$ or $\mathrm{MSH} 2$ mutation had significantly higher median family history scores than individuals with MSH6 or PMS2 mutations. This was expected given the lower lifetime risks of cancer with MSH6 and PMS2 mutations. The differences between the family history scores of the MMRD+/germ-line- group and the LS group are driven by the family history scores of individuals with an MLH1 or an MSH2 mutation, both overall and when the MMRD+/germ-line- group and the LS group are compared by gene implicated. Overall family history scores of MMRD+/ germ-line- individuals were more similar to family history scores of individuals with an MSH6 or a PMS2 mutation. This could indicate that the cancer risks for MMRD+/germ-lineindividuals are more similar to those of individuals with an MSH6 or a PMS2 mutation, as shown by Rodriguez-Soler et al. ${ }^{19}$ It could also indicate that some individuals in the MMRD+/ germ-line- group may have MMRD tumors attributable to a low-penetrant germ-line mutation in a currently unidentified cancer predisposition gene. Regarding the importance of family history, a logistic regression model showed that the likelihood of testing germ-line positive increases with the strength, or the suggestiveness, of the family history, even in this population of patients who all have abnormal tumor study results. In populations that have undergone no tumor studies, such as those utilized to design the models, the same correlation is observed. . $^{16,20}$ This further strengthens the argument that the MMRD+/germline- group is likely a heterogeneous group of MMRD tumors secondary to heritable causes and MMRD tumors secondary to sporadic causes.

We did observe a wide range of family history scores on both modalities in all four groups. This range was more pronounced with MMRpro than with PREMM ${ }_{1,2,6}$. This observation is not unexpected in the MMRD+/VUS group, because this group likely has a mix of mutations that are deleterious and mutations that are polymorphisms. It is well documented that the family history of LS is variable and, thus unsurprising, that there is a wide range of scores observed in the LS group. The sporadic MSI-H group tended to have lower family history scores; however, there were a number of outliers on both modalities. It is possible that the family history of cancer in these families is attributable to common environmental exposure, lowpenetrant genes, or a combination of the two. Finally, the fact that we observed a wide range of scores in the MMRD+/germline- group strengthens the argument that this group is likely a mixture of hereditary and sporadic, although it is impossible set an absolute cutoff for what is LS in this group because we observed individuals with a confirmed diagnosis of LS with no family history, as well as individuals in the MMRD+/germline- group with highly suggestive family histories.

As universal tumor screening protocols have become more widespread, the MMRD+/germ-line- cohort has only continued to expand. Although the body of evidence suggests that not all MMRD+/germ-line- tumors are secondary to LS, we currently have no way of distinguishing between false-positive tumor study results and true-positive tumor study results, and this must be acknowledged as a limitation of universal tumor screening strategies. In addition, clinical management of these individuals and their families becomes quite complicated. At this time, we recommend that these individuals follow the same screening guidelines as individuals with LS, because no clinical tools exist yet to distinguish LS and MMRD+/germ-line- individuals. However, it is quite possible that we are subjecting MMRD+/ germ-line-individuals and their families to unnecessary invasive screening procedures. Based on young average age of diagnosis in the probands seen in our study and the young age of cancer onset in relatives, ${ }^{19}$ it seems reasonable to begin offering cancer screenings at a younger age than recommended for the general 
population. However, the question of exactly how to screen MMRD+/germ-line- individuals and their families remains. Following these individuals with general population screening recommendations when an increased risk of cancer and an increased family history of cancer have been observed could mean we would begin missing preventable cancers. However, it is also likely inappropriate to be subjecting these individuals to increased surveillance when they do not appear to have the same cancer risks as individuals and families with LS. ${ }^{19}$ Until we are able to definitely determine who has LS and who does not, it will continue to be difficult to make appropriate screening recommendations for MMRD+/germ-line- individuals. Further work needs to be performed to further define the CRC and extracolonic cancer risks in MMRD+/germ-line- individuals in order to develop appropriate surveillance recommendations.

Recent population-based studies also support these conclusions. Rodriguez-Soler et $\mathrm{a}^{19}$ showed that families of individuals with MMRD tumors but no identifiable germ-line mutation are at lower risk for development of CRC in their lifetime than LS families but are at higher risk than individuals with a known sporadic tumor. In addition, they showed that the family histories of individuals with LS tended to be more suggestive than those with no identifiable germ-line mutation. Buchanan et $\mathrm{al}^{21}$ showed that tumor studies alone have a low positive predictive value among EC patients. There has been increasing research into other possible sporadic causes of MMRD tumors, ${ }^{13,14,21}$ with Mesencamp et a $\mathrm{l}^{15}$ showing that up to $50 \%$ of unexplained abnormal tumor study results may be attributable to biallelic somatic mutations in the MMR genes. The only clinical testing readily available, however, continues to be $M L H 1$ hypermethylation and BRAF V600E mutation analysis. Therefore, at this time we are unable to determine the number of $M M R D+/ g e r m-$ line-individuals who truly have a sporadic tumor secondary to biallelic somatic mutations. As our tumor genetic testing technology continues to improve and become more widespread, this will likely change. Many of these recent population-based studies provided some analysis of family history. However, our study is the first, to our knowledge, to provide an in-depth analysis of the family histories of individuals with abnormal tumor study results for both endometrial and colorectal tumors.

There are limitations to this study. A number of individuals in the MMRD+/germ-line- group were lost to follow-up or were deceased before a complete genetic work-up could be completed. It is possible that some of these individuals truly had LS or truly had a sporadic MSI-H tumor, but the appropriate testing was never performed. Six were lost to follow-up with no sporadic tumor work-up, and 24 only had MLH1hypermethylation performed. Despite this, the MMRD+/ germ-line- group still had less suggestive family histories than the LS group, and removing these six individuals from our data analysis did not affect the final outcome of the study (data not shown). Although rare, some individuals with LS have tumors that display $\mathrm{MLH1}$ hypermethylation, ${ }^{22}$ and there are also cases of heritable constitutional MLH1 hypermethylation. ${ }^{23}$ Therefore, we cannot exclude that there may be individuals in the MSI-H sporadic group who truly have LS or who have constitutional MLH1 hypermethylation; however, based on the rarity of these phenomena, this would be unlikely to affect the overall conclusions of the study.

Because this was not a population-based study, there is the potential for referral bias. For CRCs diagnosed or treated before 2009, the practice at the MD Anderson Cancer Center was to perform tumor studies and/or provide a genetics referral only for "high-risk" individuals. After 2009, the MD Anderson Cancer Center adopted a more universal approach to tumor studies for CRCs. Tumor studies for EC, however, have only been routinely performed at the MD Anderson Cancer Center since August 2012. Regardless of this potential bias, referred MMRD+/germline- individuals still had less suggestive family histories than LS individuals. It is possible that the schism between family history scores for MMRD+/germ-line- and LS individuals could be more pronounced in the general population.

Possible future directions from this study include sequencing the tumor DNA of all the individuals in the MMRD+/germline- group to determine how many have biallelic somatic MMR mutations. The family histories of those with biallelic somatic MMR mutations and those whose MMRD tumors continue to be unexplained could then be compared. This could further strengthen the argument presented here.

In conclusion, MMRD+/germ-line- individuals have a less suggestive family history than individuals with LS but a more suggestive family history than individuals with a sporadic MSI-H tumor, and a family history similar to that of individuals with MMRD+/VUS. These results further reinforce the need to continue exploring other causes of MMRD tumors, because they do not all appear to be classical LS. As our understanding of other somatic and epigenetic causes of MMRD tumors expands, we need to reevaluate our current testing practices and develop other clinical testing to rule out all known somatic and epigenetic causes. We may also need to reconsider the current screening guidelines for MMRD+/germ-line-individuals, because we may be subjecting these individuals to unnecessary invasive surveillance.

\section{ACKNOWLEDGMENT}

This research was supported in part by the National Institutes of Health through MD Anderson Cancer Center support grant CA016672.

\section{DISCLOSURE}

The authors declare no conflict of interest.

\section{REFERENCES}

1. Lynch HT, Smyrk TC, Watson P, et al. Genetics, natural history, tumor spectrum, and pathology of hereditary nonpolyposis colorectal cancer: an updated review. Gastroenterology 1993;104:1535-1549.

2. Ponti G, Losi L, Pedroni M, et al. Value of MLH1 and MSH2 mutations in the appearance of Muir-Torre syndrome phenotype in HNPCC patients presenting sebaceous gland tumors or keratoacanthomas. J Invest Dermatol 2006;126:2302-2307.

3. South CD, Hampel H, Comeras I, Westman JA, Frankel WL, de la Chapelle A. The frequency of Muir-Torre syndrome among Lynch syndrome families. J Natl Cancer Inst 2008;100:277-281. 
4. Watson P, Vasen HF, Mecklin JP, et al. The risk of extra-colonic, extra-endometrial cancer in the Lynch syndrome. Int J Cancer 2008;123:444-449.

5. Barrow $E$, Robinson $L$, Alduaij $W$, et al. Cumulative lifetime incidence of extracolonic cancers in Lynch syndrome: a report of 121 families with proven mutations. Clin Genet 2009;75:141-149.

6. Evaluation of Genomic Applications in Practice and Prevention (EGAPP) Working Group. Recommendations from the EGAPP Working Group: genetic testing strategies in newly diagnosed individuals with colorectal cancer aimed at reducing morbidity and mortality from Lynch syndrome in relatives. Genet Med 2009;11:35-41.

7. Hampel $\mathrm{H}$, Frankel $\mathrm{WL}$, Martin E, et al. Feasibility of screening for Lynch syndrome among patients with colorectal cancer. J Clin Oncol 2008;26: 5783-5788.

8. Fishel $\mathrm{R}$, Kolodner RD. Identification of mismatch repair genes and their role in the development of cancer. Curr Opin Genet Dev 1995:5:382-395.

9. Ligtenberg MJ, Kuiper RP, Chan TL, et al. Heritable somatic methylation and inactivation of MSH2 in families with Lynch syndrome due to deletion of the 3' exons of TACSTD1. Nat Genet 2009;41:112-117.

10. Moreira L, Balaguer F, Lindor N, et al.; EPICOLON Consortium. Identification of Lynch syndrome among patients with colorectal cancer. JAMA 2012;308: 1555-1565.

11. Heald B, Plesec T, Liu X, et al. Implementation of universal microsatellite instability and immunohistochemistry screening for diagnosing lynch syndrome in a large academic medical center. J Clin Oncol 2013:31:1336-1340

12. Morak M, Koehler U, Schackert HK, et al.; German HNPCC consortium. Biallelic MLH1 SNP CDNA expression or constitutional promoter methylation can hide genomic rearrangements causing Lynch syndrome. J Med Genet 2011;48: 513-519.

13. Cancer Genome Atlas Network. Comprehensive molecular characterization of human colon and rectal cancer. Nature 2012:187:330-337.
14. Sourrouille I, Coulet $F$, Lefevre $J H$, et al. Somatic mosaicism and double somatic hits can lead to MSI colorectal tumors. Fam Cancer 2013;12:27-33.

15. Mesenkamp AR, Vogelaar IP, van Zelst-Sstams WAG, et al. Somatic mutations in MLH1 and MSH2 are a frequent cause of mismatch-repair deficiency in Lynch Syndrome-like tumors. Gastroenterology 2014;146:643-646.

16. Chen S, Wang W, Lee $S$, et al.; Colon Cancer Family Registry. Prediction of germline mutations and cancer risk in the Lynch syndrome. JAMA 2006;296:1479-1487.

17. Howlander N, Noone AM, Krapcho M, et al. (eds). SEER Cancer Statistics Review, 1975-2009 (Vintage 2009 Populations). 2012. http://seer.cancer.gov/ csr/1975_2009_pops09/. Accessed 1 March 2012.

18. Stoffel E, Mukherjee B, Raymond VM, et al. Calculation of risk of colorectal and endometrial cancer among patients with Lynch syndrome. Gastroenterology 2009;137:1621-1627.

19. Rodríguez-Soler M, Pérez-Carbonell L, Guarinos C, et al. Risk of cancer in cases of suspected lynch syndrome without germline mutation. Gastroenterology 2013;144:926-932.e1; quiz e13.

20. Kastrinos F, Steyerberg EW, Mercado R, et al. The PREMM $(1,2,6)$ model predicts risk of $\mathrm{MLH1}, \mathrm{MSH} 2$, and $\mathrm{MSH} 6$ germline mutations based on cancer history. Gastroenterology 2011;140:73-81.

21. Buchanan DD, Tan YY, Walsh MD, et al. Tumor mismatch repair immunohistochemistry and DNA MLH1 methylation testing of patients with endometrial cancer diagnosed at age younger than 60 years optimizes triage for population-level germline mismatch repair gene mutation testing. J Clin Oncol 2014:32:90-100.

22. Rahner N, Friedrichs N, Steinke $V$, et al. Coexisting somatic promoter hypermethylation and pathogenic MLH1 germline mutation in Lynch syndrome. J Pathol 2008;214:10-16.

23. Niessen RC, Hofstra RM, Westers $\mathrm{H}$, et al. Germline hypermethylation of MLH1 and EPCAM deletions are a frequent cause of Lynch syndrome. Genes Chromosomes Cancer 2009:48:737-744. 